Asian Journal of Computer Science and Technology

ISSN: 2249-0701 Vol.9 No.2, 2020, pp. 18-23

(C) The Research Publication, www.trp.org.in

\title{
Application of IPF to Achieve CSR Routing in Adhoc Networks
}

\author{
B.P. Sreejith Vignesh \\ Department of Information Technology, Sri Krishna Adithya College of Arts and Science, Coimbatore Tamil Nadu, India \\ Email: authorsree@gmail.com
}

\begin{abstract}
Wireless sensor network (WSN) comprises of sensor nodes that requires resource optimized routing techniques for efficient data collection. Since data collection consumes more energy, it is of great importance to design resource optimized routing to achieve both resource and routing efficiency together. In this work, a resource optimized routing method called Channel State Responsive Routing protocol using Intensity Probable Field (CSRR-IPF) is proposed. CSRR-IPF minimizes energy consumption and prolong network lifetime using Intensity Probable Field based on channel state. The goal of this Intensity Probable Field is to make efficient data collection at the sink node at the same time to achieve efficient routing among neighbors. The Intensity Probable Resource Optimized model limits the node movements by maximizing the minimum energy cost so that the energy of nodes in the entire network is balanced and therefore prolonging the network lifetime. With the Intensity Probable Resource Optimized model, Channel State Responsive Routing Protocol is designed that aggregates the collected data improving the routing efficiency. The performance of the proposed CSRRIPF is evaluated in the context of energy consumption, network lifetime and routing time with respect to per node density and per data packet basis. The research results are analyzed and benchmarked against the state-of-the-art methods. Simulation results show that the algorithm show a significant improvement and extends the network lifetime, has good performance on energy balance of sensors, and prolongs the network lifetime compared with similar algorithms.

Keywords: Adhoc Networks, Wireless Sensor Network, Data Collection, Channel State, Responsive Routing, Intensity Probable.
\end{abstract}

\section{INTRODUCTION}

As multiple sensor nodes in WSN come into play, efficient management of relay nodes for data collection at the sink node becomes a critical issue for improving the system performance. Channel estimation and performing routing accordingly results in the significant improvement in ad hoc networks. Several research works has been conducted in this area.

In [1], joint stable routing and channel assignment was performed for mobile cognitive ad hoc networks by using integrated transmission cost and relay workload resulting in the optimized routing. An energy-efficient reliable routing was designed in [2] based on the energy cost and minimum energy and lifetime of the network. However, without consideration of channel state and resource allocation, routing gets compromised. To address this issue, resource allocation with flexible channel cooperation through optimization solution based on Nash bargaining systems was designed in [3]. Wireless adhoc communication systems in cooperative structure provide higher potential gains in the transmission rate of the wireless link. In [4], a subcarrier based resource allocation was designed with the objective of reducing the computational complexity during routing. A robust uplink resource allocation model was studied in [5] based on perfect channel state information resulting in the improvement of computational complexity. Assessing performance gains through global resource control was studied in [6]. A two step approach using mixed model and resource allocation was designed in [7] based on the uplink and downlink sharing resulted in the minimization of computational complexity.

With the increasing growth in the portable wireless communication devices, one of the most challenging question lies in whether efficient harness of computation is said to occur or not. In [8], randomized network structuring and packet routing framework was presented based on nearest neighbor communications resulting in the improvement of energy consumed per packet. In [9], resource allocation with multiple relays was designed with the aid of greedy algorithm that resulted in the improvement of end to end rate. One of the main issues in Workflow Management System is the proper and optimal allocation of resources. In [10], discrete optimization was applied to reduce the computational complexity and system workload was designed.

In this work, we have introduced a Channel State Responsive Routing protocol using Intensity Probable Field (CSRR-IPF) method. Here, the data collection is performed by the sink node by calculating minimum hop count and probable value for respective sensor nodes in a dynamic manner. Followed by this, a channel state is measured to continue with the process of routing. This calculation is performed through optimal location of sink node which depends on channel state.

The remaining part of this paper is organized as follows. Section 2 briefly reviews related work. Section 3 presents the network model, formalizes the CSRR-IPF problem and proposes a channel state method. Systematical examination by testing and evaluation is performed in Section 4 using NS2-based simulation system. Section 5 concludes this paper. 


\section{RELATED WORKS}

Resource allocation in wireless sensor networks has been investigated by several resource persons. In [11], a polynomial time solution subject to user's channel conditions for efficient resource allocation was performed based on the total amount of resource available in the network. Another efficient routing method by employing forwarding zones and routing zones was presented in [12] to obtain optimal energy consumption during routing.

The two basic requirements of WSN are low delay and high data integrity resulting in delay sensitive and high integrity applications. With light load, both delay sensitive and high integrity are easily addressed. However, to address the issue with heavily loaded network, in [13], a virtual hybrid potential field was designed called, Integrity and Delay Differentiated Routing. In [14], joint admission control and routing with the objective of addressing the issues related to routing and network lifetime as investigated. A reinforcement learning scheme was utilized in [15] to provide a stochastic adaptive opportunistic routing scheme, resulting in high throughput rate.

Link stability and Energy aware routing was presented in [16] to solve bi-objective optimization formulation resulting in the improvement in the average energy consumption and network lifetime. In [17], joint distributed channelization and routing was presented using cognitive spread spectrum channelization formulation. In [18], Dynamic Priority Resource Allocation (DPRA) for fair scheduling in wireless communication system was presented that resulted in the improvement of system throughput.

As technology improves and scales down to the nano-scale integration of billions of transistors into a single chip has become more common. In [19], Traffic Balancing Oblivious Routing (TBOR) was designed to reduce the average delay during routing. However, with sparse network, average delay increases proportionately. Geographic and opportunistic routing with Depth Adjustment based topology control for communication Recovery (GEDAR) over void regions was presented in [20] for sparse network.

To attain resource optimized routing in wireless sensor network, in this paper, we propose a channel state mechanism to optimize the resources during data collection at the sink node by balancing energy consumption of each sensor node with the consideration about the network lifetime.

Different from the classical resource optimized routing algorithm working with the joint stable routing and channel assignment to achieve energy efficiency and network lifetime, the reported algorithm here is constructed according to the channel availability that determines the minimum total energy cost and hop between the source and destination nodes to achieve maximum lifetime of the whole network and improve resource efficiency.

\section{METHODOLOGY}

In this work, we specifically address the issue of WSN deployment under resource optimization and efficient routing, called, Channel State Responsive Routing protocol using Intensity Probable Field (CSRR-IPF). The CSRR-IPF presents a new resource optimized routing method based on Channel State. We first start with the design of network model, followed by the problem formulation and finally the proposed CSRR-IPF method.

\section{A. Network Model}

In wireless sensor networks, the sensor nodes sense the environment in a periodical manner and perform data collection at the sink node. In order to describe a resource optimized routing algorithm more clearly, we define wireless sensor networks and neighbors who sent data packets ' $D P=D P_{1}, D P_{2}, \ldots, D P_{n}$ ' to and fro. Let us consider a wireless sensor network expressed as an undirected graph ' $G(V, E)$ ', where ' $V$ ', the vertices represents the set of all nodes and edges ' $E$ ' represents the links between nodes. Then, the vertices are formulated as given below.

$$
' V=V_{s n} \cup V_{s}^{\prime} \ldots \ldots \ldots \ldots \ldots
$$

From (1), ' $V_{s n}$ ' represents the sensor nodes and ' $V_{s}$ ' represents the sink nodes respectively. The edges are then formulated as given below.

$$
E=\left\{(p, q)\left|p, q \in V_{s n}\right| p \in V_{s n}, q \in V_{s}\right\}, \operatorname{Dis}(p, q)<R
$$$$
\text { .........(2) }
$$

From (2), ' $\operatorname{Dis}(p, q)$ ' denotes the distance between the sensor nodes ' $p$ and $q$ '.

\section{B. Problem Formulation}

The objective of this work is to identify a feasible data collection method with resource optimized routing algorithm, such that all the individual data packets originated from each sensor node ' $V_{s n}$ ' is collected at the sink node ' $V$ ' ' based on the channel condition. The total resource optimized routing problem is then expressed as a joint optimization problem that not only optimizes the resource (i.e. energy consumption and network lifetime) but also improves routing during data collection in WSN.

\section{Intensity Probable Resource Optimized Model}

The Intensity Probable Resource Optimized (IPRO) model in the CSRR-IPF method aims to ensure data packets to be collected at a fair rate at the sink node. Figure 1 shows an example of IPRO algorithm. In Figure 1, sensor node ' $s n_{p}$ ' and ' $s n_{q}$ ' has data packet to be sent to the sink node, where data collection is said to be performed at the sink node. Since it has three neighbors, there are three alternative paths to the sink node. The CSRR-IPF uses three values, the 
minimum hop count, probable value in intensity probable field and energy cost of each sensor ' $I(s n), P_{i}(p), E C$ ', so as to make routing decisions, ensuring resource optimization (energy efficiency and network lifetime).

Let ' $H(p, q)$ ' represents the hop count of sensor ' $s n \in V_{s n}$ ', to sink ' $V_{s}$ '. When the hop counts to all the sinks are identified, the minimum hop count is assigned as the intensity of sensors, and is expressed as given below.

$$
I(s n)=\operatorname{MIN}\left(H(p, q) \mid p \in V_{s n}, q \in V_{s}\right)
$$

With the objective of optimizing the resource during data packets collection at the sink node, the CSRR-IPF method measures the difference between the total sensor nodes in network and intensity of sensor node ' $p$ ' and is expressed as below.

$$
P_{i}(p)=S N-I(p)
$$

From (4), ' $P_{i}(p)$ ' measures the probable value in intensity probable field ' $I(p)$ '. With the probable value, the force ' $F_{i}$ ' between two sensor node ' $s n_{i}$ ' and ' $s n_{j}$ ' is expressed as given below.

$$
F_{i}\left(s n_{i}, s n_{j}\right)=\frac{P_{i}\left(s n_{i}\right)-P_{i}\left(s n_{j}\right)}{\operatorname{Cost}\left(s n_{i}, s n_{j}\right)}
$$

From (5), the force is obtained through the ratio of difference between the probable value between two sensor nodes and ' $\operatorname{Cost}()^{\text {', }}$ denotes the energy cost of the sensor nodes ' $s n_{i}$ ' and ' $s n_{j}$ ' respectively.

With Intensity Probable Field, data collection is performed at the sink node along the shortest path with minimum hop count, which realizes the resource optimization (i.e. energy efficiency among sensors). In addition, the energy efficient model is enhanced to limit the node movements so that the energy of sensor nodes in the whole WSNs is said to be balanced, improving the network lifetime.

An imbalanced energy cost of the network results in out of energy, entirely changing the structure of WSN, compromising the lifetime of the network. Therefore, in the CSRR-IPF method, to prolong the network lifetime, the energy cost is minimized during data packet collection at the sink node and is as expressed below.

$$
E C=\operatorname{MIN}\left(I E_{p}-E_{p}\right) * \operatorname{Mag}_{p} \ldots \ldots(6)
$$

From (6), the energy cost is obtained by the product of the difference between the initial energy of node ' $I E_{p}$ ' and energy of node ' $E_{p}$ ' and the distance of node ' $p$ ' that moves in each magnitude ' $M a g_{p}$ '. Figure 2 shows Intensity Probable Resource Optimized (IPRO) algorithm.

For efficient data collection at the sink node, the IPRO algorithm in the CSRR-IPF method initially measures the hop count. With the measured hop count, in the intensity probable field, the probable value is obtained. With this probable value, force between two sensor nodes is measured to optimize the energy consumption. Further, the IPRO algorithm, prolong the network lifetime by maximizing the minimum energy cost.

\section{Channel State Responsive Routing Protocol Using Intensity Probable Field}

Once resource optimized model has been designed, suitable energy optimized nodes are identified through IPRO algorithm as provided in figure. The energy threshold level is more significant than others. On this note, IPRO algorithm is therefore aimed to maximize the minimum energy between the nodes and to save energy during data collection at the sink node using Channel State Responsive Routing Protocol. Figure 2 shows the flow diagram of Channel State Responsive Routing Protocol.

The Channel State Responsive Routing Protocol in the CSRR-IPF method is designed on the basis of the channel state. In the Channel State Responsive Routing Protocol, we proceed with the assumption that the sink node collects the data packets from sensor nodes only when the channel state is idle. On contrary, if the channel state is busy, the sink node does not collect the data packets from the sensor nodes.

The sensor nodes in the CSRR-IPF send a probe packet to identify the channel state information. Figure 4 shows the structure of Channel State Responsive Routing Protocol (CSRRP).

\begin{tabular}{|c|c|c|c|}
\hline $\begin{array}{c}\text { Sender } \\
\text { node ' } s n_{i} \text { ' }\end{array}$ & $\begin{array}{c}\text { Node Type } \\
\text { ' } N T_{M} \text { ' }\end{array}$ & $\begin{array}{c}\text { Energy } \\
\text { ' } E_{s n} \text { ' }\end{array}$ & $\begin{array}{c}\text { Channel state } \\
\text { ' } C S=0 / 1\end{array}$ \\
\hline
\end{tabular}

Fig. 1 Structure of Channel State Responsive Routing Protocol

As shown in the figure, the CSRR-IPF includes the information about the sender node ' $s n_{i}$ ', the node type, where ' $N T_{M}=0$ ' or ' $N T_{M}=1$ '. In case of ' $N T_{M}=0$ ' implies the sink node whereas ' $N T_{M}=1$ 'implies a normal neighbor node. ' $E_{S n}$ ' symbolizes the energy of the sender node and finally, the channel state is obtained through ' $C S$ ' where ' $C S=0$ ' refers to the idle state and ' $C S=1$ ' refers to the busy state.

Data collection from sensor nodes to the sink node is performed only when the channel state is idle, where the data packets are collected at the sink node. With the identified channel state, the CSRR-IPF measures the optimal location of sink node. The optimal location of sink node with respect to minimum energy consumption and maximum network lifetime is expressed as given below.

$\left(p_{0}, q_{0}\right) \operatorname{argmin}\left(\max \left(\sqrt{\left(p-p_{i}\right)^{2}+\left(q-q_{i}\right)^{2}}\right)\right)$.

From (7), ' $\left(p_{0}, q_{0}\right)$ ' symbolizes the optimal location of sink node where data collection is performed which is equivalent 
to minimizing the maximum distance between the sink node and the sensor node respectively. With the energy consumption ' $E C_{s n}$ ' and minimum hop ' $H$ ', the channel state responsive route 'CSRR' is expressed as given below.

$$
\begin{aligned}
& E C_{s n}=\operatorname{MIN}\left(I E_{p}-E_{p}\right) \\
& H=\operatorname{Min}(H(p, q)) \ldots \ldots \ldots \\
& C S R R=E C_{S n} * H \ldots \ldots \ldots \ldots
\end{aligned}
$$

With the optimal location, Channel State Responsive Routing ' $C S R R$ ' is evaluated on the basis of the energy consumption and number of hops as given above. Figure 5 shows the Channel State Responsive Routing algorithm.

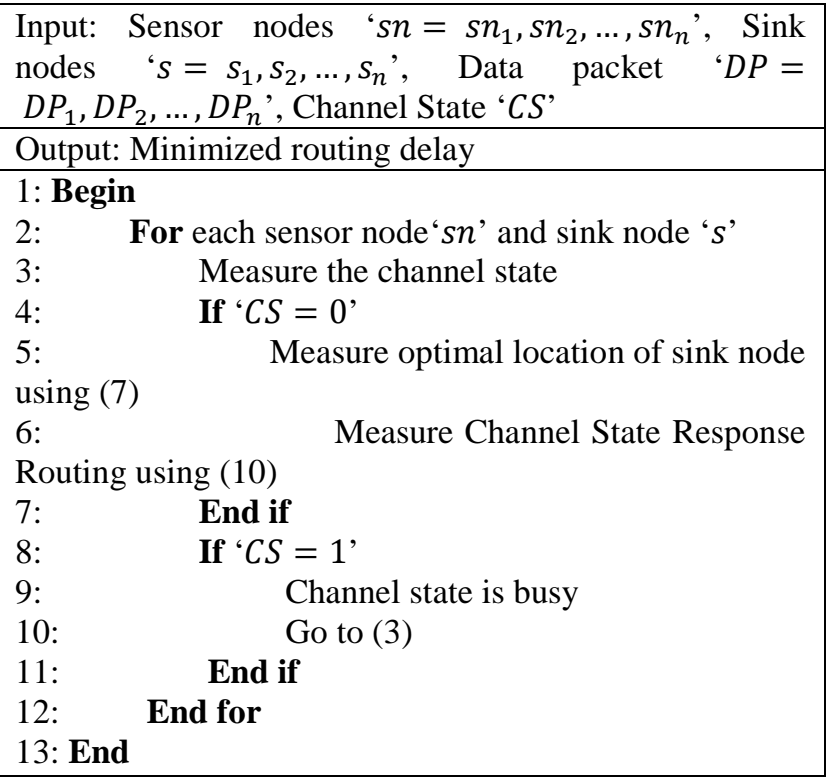

Fig. 2 Channel State Responsive Routing algorithm

As shown in the figure, a new routing algorithm based on channel state in WSN is presented, in which the sensor nodes are distributed in a network. The sensed data is collected based on the optimized resource in its range and then forwards them to a sink node. The CSRR-IPF though reduced the energy consumption during data collection and prolong the network lifetime, but routing overhead at the sink node increases with the increased aggregation rate. For that reason, to reduce the routing overhead, an additional channel state response routing algorithm is designed in which the sensed data is gathered based on the channel state response and act according to the busy or idle state. This in turn reduces the routing overhead and therefore improving the routing efficiency.

\section{RESULTS AND DISCUSSION}

In this section, the performance of the proposed Channel State Responsive Routing protocol using Intensity Probable Field (CSRR-IPF) is evaluated via NS2. We calculate the energy consumption for data collection at the sink node. We define the network lifetime as the network that minimizes the maximum sensor node load, which is counted by round.
We compare the performance of CSRR-IPF method with Joint Stable Routing and Channel Assignment (J-SRCA) [1] and Energy Efficient Routing Algorithm [2] on the network lifetime, energy consumption and routing efficiency through average routing delay time. In our simulations, sensors are randomly and uniformly deployed over the square monitoring area. Sinks are uniformly distributed at the outside of the monitoring area. Other simulation parameters are given in Table I.

\section{TABLE I SIMULATION PARAMETERS}

\begin{tabular}{|l|c|}
\hline Parameter & Value \\
\hline Network coverage & $1400 \mathrm{~m} * 1400 \mathrm{~m}$ \\
\hline Node density & 70 \\
\hline Number of sinks & $1-5$ \\
\hline Initial energy & $0.5 \mathrm{~J}$ \\
\hline Number of packets sent & $7-49$ \\
\hline Maximum transmission range & $50 \mathrm{~m}$ \\
\hline
\end{tabular}

\section{A. Network Lifetime}

Network lifetime in WSN is the time until the first sensor node or group of sensor nodes runs out of energy. Therefore, the network that minimizes the maximum sensor node load is the one that will ensure the maximum network lifetime and this is achieved using the proposed LPAHC framework.

TABLE II NETWORK LIFETIME

\begin{tabular}{|l|c|}
\hline Methods & Network lifetime (ms) \\
\hline CSRR-IPF & 88.21 \\
\hline J-SRCA & 80.32 \\
\hline EERA & 73.23 \\
\hline
\end{tabular}

As listed in table II, the CSRR-IPF method, J-SRCA and EERA measures the network lifetime which is measured in terms of milliseconds (ms). The network lifetime using CSRR-IPF method offers comparable values than the stateof-the-art methods.

Figure 6 gives the network lifetime with different routing algorithms when the number of sinks changes from 1 to 5 with a node density of 70 . It can be seen from the figure that CSRR-IPF has extended the network lifetime compared with J-SRCA and EERA. J-SRCA only considers the quality of links when making routing decisions.

The routing path is constructed by only considering the link quality that has adverse effect with the increase in the network size and results in serious impact on network lifetime.

J-SRCA has shorter network lifetime found to be higher than EERA but lower than CSRR-IPF with the increase of the number of sinks. 


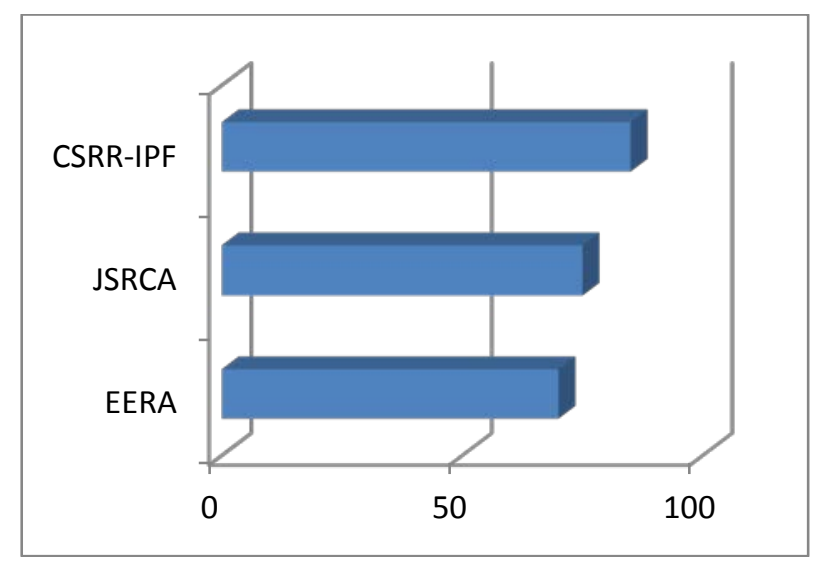

Fig. 3 Network lifetime

EERA has considered minimum energy cost and minimum energy in routing, but more energy is consumed in the process to obtain the energy level of the path and have adverse impact on varying conditions. In EERA, minimum energy cost routing and reliable minimum energy routing are considered when making routing decisions. But the uneven energy consumption will affect energy balance.

In CSRR-IPF, we use Intensity Probable Field with minimum hop count to limit the node movement and select the node with good energy balance effect as next hop. So its network lifetime is longer than J-SRCA and EERA with 19\% improvement compared to J-SRCA and 9\% compared to EERA.

\section{B. Energy Consumption}

Energy consumption for data collection is the product of energy consumed by a single sensor node and the total sensor nodes in WSN.

$$
E C=\text { Energy }_{S N} * \text { Total }_{S N}
$$

From (11), ' $E C$ ' is the energy consumption whereas ' $S N$ ' represents the sensor nodes, with the consumption of energy measured in terms of Joules. Figure 7 shows the energy consumption during data collection at the sink node for different number of nodes (i.e. node density). As the number of nodes increases, the performance improvement of the proposed technique is in par with J-SRCA and EERA showing a small marginal average improvement of $8 \%$ compared to J-SRCA and 15\% compared to EERA. However, both J-SRCA and EERA and proposed CSRRIPF method show significant decrease in network lifetime as the number of nodes is increased (from 30 to 40). This resulted in a decrease of network lifetime from $9 \%$ to $7 \%$ and $19 \%$ to $15 \%$ respectively when compared to J-SRCA and EERA.

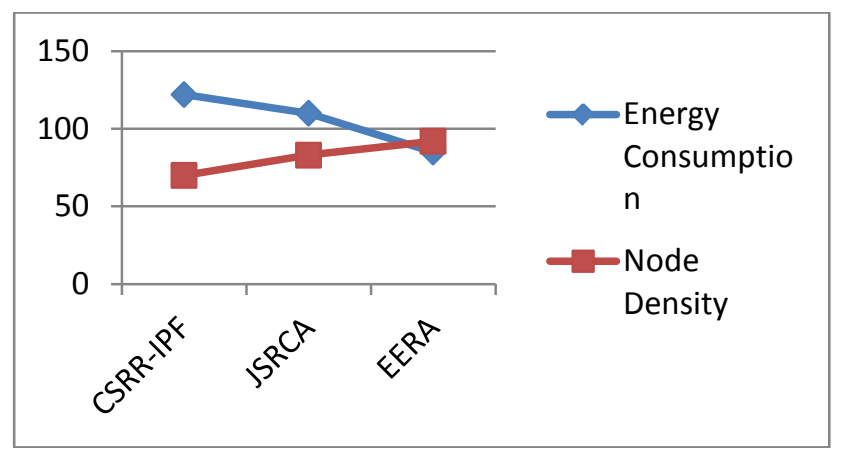

Fig. 4 Energy Consumption

According to Figure 4 we can conclude that the total energy consumption for data collection at the sink node which was found by CSRR-IPF method is smaller than the total energy consumption for data collection at the sink node which was found by J-SRCA and EERA, this stems from two respect reasons commonly. Firstly, this is because IPRO algorithm adopts the strategy of combination optimal, minimum hop count, probable value in intensity probable field, energy cost of each sensor, this way can improve the success rate of data collection at the sink node, hence put down the total energy consumption.

Secondly, IPRO algorithm uses the probable value in intensity probable field for the optimal data collection and lets the sensor nodes to travel along the optimal route to complete data collection. This strategy can put down the workload of data collection hence reduce the total energy cost.

\section{Average Routing Delay Time}

The average routing delay time is the time taken to collect the data packets from the sensor node and sent to the sink node in WSN. It is measured in terms of milliseconds (ms) and is formulated as given below.

$A R D T=$ Time $\sum_{i=1}^{n} D C\left(D P_{i}\right)$

From (12) ' $A R D T$ ' refers to the average routing delay time for collection of data packets ' $D P_{i}$ ' to the sink node in WSN.

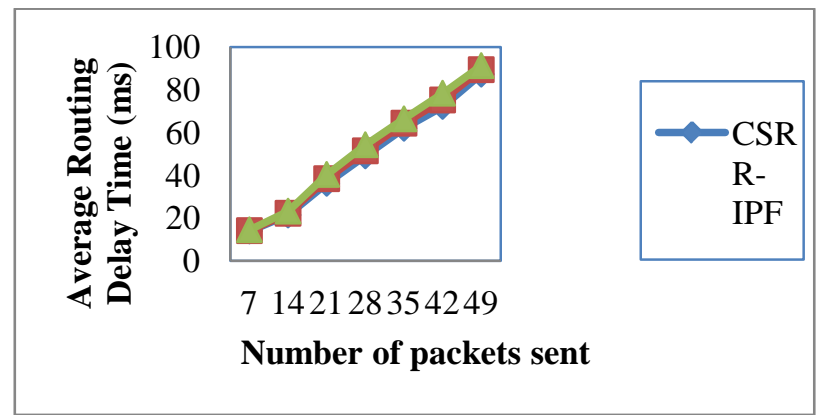

Fig. 5 Average Routing Delay Time 
Figure 5 shows the relationships of the average routing delay time with respect to varying number of packets sent. According to Figure 8, it can be seen that the minimal average routing delay time found by CSRR-IPF is lower than the minimal average routing delay time in optimal routes which were found by J-SRCA and EERA. This is because the CSRR-IPF method designed a new optimal route evaluation standard based on the channel state and uses this rule to evaluate the performance of the optimal route. This evaluation standard obtains the optimal location of sink node with less energy in the process of searching route, so the minimal energy of the nodes in optimal route is the largest. So the average routing delay time found by CSRR-IPF is the smallest one. It was observed to be $4 \%$ reduced when compared to J-SRCA and 8\% reduced when compared to EERA.

\section{CONCLUSION}

In these research findings, an Intensity Probable Field is applied to achieve Channel State Responsive Routing protocol in Adhoc networks using for efficient data collection at the sink node is presented. In CSRR-IPF, data collection is performed and data packets are collected at the sink node based on the intensity probable field and energy cost of the sensor nodes with dynamically computed probability called as probable value. The probable value is calculated based on the total sensor nodes in the network and the intensity of the sensor nodes. Next, a channel state responsive routing protocol is designed and investigated via channel state through which the optimal location of sink node is measured. The performance of the CSRR-IPF method has been compared against J-SRCA and EERA. Simulation results showed that CSRR-IPF method performs better than other representative energy efficient routing in terms of energy consumption, network lifetime and average routing delay time.

\section{REFERENCES}

[1] Feilong Tang, Minyi Guo, Song Guo, and Cheng-Zhong Xu, "Mobility Prediction Based Joint Stable Routing and Channel Assignment for Mobile Ad Hoc Cognitive Networks", IEEE Transactions on Mobile Computing, Vol.5, No.8, July 2015, pp. 1-11.

[2] Javad Vazifehdan, R. Venkatesha Prasad, and Ignas Niemegeers, "Energy-Efficient Reliable Routing Considering Residual Energy in Wireless Ad Hoc Networks”, IEEE Transactions on Mobile Computing, Vol.13, No.2, February 2014, pp. 434-447.

[3] Hong Xu, Baochun Li, "Resource Allocation with Flexible Channel Cooperation in Cognitive Radio Networks", IEEE Transactions on Mobile Computing, Vol.12, No. 5, May 2013, pp.957 - 970.

[4] Hanan Al-Tous and Imad Barhumi, "Resource Allocation for Multiple-Sources Single-Relay Cooperative Communication OFDMA Systems”, IEEE Transactions on Mobile Computing, Vol. 15, No. 4, April 2016, pp 964-981.

[5] Nader Mokari, Saeedeh Parsaeefard, Paeiz Azmi, and Hamid Saeedi, "Robust Ergodic Uplink Resource Allocation in Underlay OFDMA Cognitive Radio Networks", IEEE Transactions on Mobile Computing, Vol.15, No.2, February 2016, pp. 419-431.

[6] Rahul Amin, Jim Martin, "Assessing Performance Gains Through Global Resource Control of Heterogeneous Wireless Networks", IEEE Transactions on Mobile Computing, Vol.15, No. 2, February 2016, pp.292-305.
[7] Huan Tang, Zhi Ding, "Mixed Mode Transmission and Resource Allocation for D2D Communication”, IEEE Transactions on Wireless Communications, Vol.15, No.1, January 2016, pp.162-175.

[8] Behnam A. Rezaei, Nima Sarshar, and Vwani P. Roychowdhury, "Distributed Resource Sharing in Low-Latency Wireless Ad Hoc Networks", IEEE/ACM Transactions on Networking, Vol. 18, No.1, February 2010, pp.190-201.

[9] Ka gan Bakano glu, Stefano Tomasin, and Elza Erkip, "Resource Allocation for the Parallel Relay Channel with Multiple Relays", IEEE Transactions on Wireless Communications, Vol.10, No. 3, March 2011, pp. 792-801.

[10] Pavlos Delias, Anastasios D. Doulamis, Nikolaos D. Doulamis, and Nikolaos Matsatsinis, "Optimizing Resource Conflicts in Workflow Management Systems”, IEEE Transactions on Knowledge and Data Engineering, Vol. 23, No. 3, March 2011, pp.417-432.

[11] Wen-Hsing Kuo, Wanjiun Liao, and Tehuang Liu, "Adaptive Resource Allocation for Layer-Encoded IPTV Multicasting in IEEE 802.16 WiMAX Wireless Networks", IEEE Transactions on Multimedia, Vol.13, No.1, February 2011, pp. 116-124.

[12] Shouling Ji, Student Member, Mingyuan Yan, Raheem Beyah, and Zhipeng Cai, "Semi-Structure Routing and Analytical Frameworks for Cognitive Radio Networks", IEEE Transactions on Mobile Computing, Vol.15, No. 4, April 2016, pp. 996-1008.

[13] Jiao Zhang, Fengyuan Ren, Shan Gao, Hongkun Yang, and Chuang Lin, "Dynamic Routing for Data Integrity and Delay Differentiated Services in Wireless Sensor Networks", IEEE Transactions on Mobile Computing, Vol. 14, No.2, February 2015, pp. 328-343.

[14] Shiying Zhang, F. Richard Yu, and Victor C. M. Leung, "Joint Connection Admission Control and Routing in IEEE 802.16-Based Mesh Networks",IEEE Transactions on Wireless Communications, Vol. 9, No. 4, April 2010, pp. 1370-1379.

[15] A.A. Bhorkar, M. Naghshvar, T. Javidi, and B.D. Rao, "An Adaptive Opportunistic Routing Scheme For Wireless Ad-Hoc Networks", IEEE/ACM Transactions On Networking, Vol. 20, No. 1, Feb. 2012, pp.243 - 256.

[16] Floriano De Rango, Francesca Guerriero, , and Peppino Fazio, LinkStability and Energy Aware Routing Link-Stability and Energy Aware Routing Protocol in Distributed Wireless Networks”, IEEE Transactions On Parallel And Distributed Systems, Vol. 23, No.4, April 2012, pp. 713-726.

[17] Lei Ding, Kanke Gao, Tommaso Melodia, Stella N. Batalama, Dimitris A. Pados, And John D. Matyjas, "All-Spectrum Cognitive Networking Through Joint Distributed Channelization And Routing”, IEEE Transactions On Wireless Communications, Vol.12, No.11, November 2013, pp. 5394-5405.

[18] Chih-Ming Yen, Chung-Ju Chang, Fellow, Fang-Ching Ren, And Jian-Ann Lai, "Dynamic Priority Resource Allocation For Uplinks In IEEE 802.16 Wireless Communication Systems", IEEE Transactions On Vehicular Technology, Vol.58, No. 8, October 2009, pp.45874597.

[19] Pengju Ren, Michel A. Kinsy, And Nanning Zheng, Fellow, "FaultAware Load-Balancing Routing For 2D-Mesh And Torus On-Chip Network Topologies", IEEE Transactions On Computers, Vol.65, No. 3, March 1 2016, pp. $873-887$.

[20] Rodolfo W.L. Coutinho, Azzedine Boukerche, Luiz F.M. Vieira, and Antonio A.F. Loureiro, "Geographic and Opportunistic Routing for Underwater Sensor Networks”, IEEE Transactions on Computers, Vol. 65, No. 2, Feb 2016, pp.548-561. 